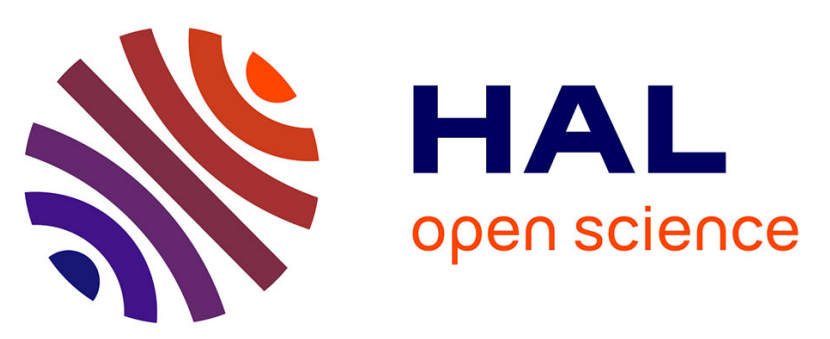

\title{
Cantilever Anemometer Based on a Superconducting Micro-resonator: Application to Superfluid Turbulence
}

\author{
Julien Salort, Alessandro Monfardini, Philippe-Emmanuel Roche
}

\section{To cite this version:}

Julien Salort, Alessandro Monfardini, Philippe-Emmanuel Roche. Cantilever Anemometer Based on a Superconducting Micro-resonator: Application to Superfluid Turbulence. Review of Scientific Instruments, 2012, 83, pp.125002. 10.1063/1.4770119 . hal-00796196

\section{HAL Id: hal-00796196 https://hal.science/hal-00796196}

Submitted on 1 Mar 2013

HAL is a multi-disciplinary open access archive for the deposit and dissemination of scientific research documents, whether they are published or not. The documents may come from teaching and research institutions in France or abroad, or from public or private research centers.
L'archive ouverte pluridisciplinaire HAL, est destinée au dépôt et à la diffusion de documents scientifiques de niveau recherche, publiés ou non, émanant des établissements d'enseignement et de recherche français ou étrangers, des laboratoires publics ou privés. 


\title{
Cantilever Anemometer Based on a Superconducting Micro-resonator: Application to Superfluid Turbulence
}

\author{
J. Salort, ${ }^{*}$ A. Monfardini, and P.-E. Roche \\ Institut Néel, CNRS/UJF - 25 rue des Martyrs, BP 166, 38042 Grenoble cedex 9, FR
}

(Dated: January 5, 2013)

\begin{abstract}
We present a new type of cryogenic local velocity probe that operates in liquid helium $(1 \mathrm{~K}<$ $T<4.2 \mathrm{~K}$ ) and achieves a spatial resolution of $\approx 0.1 \mathrm{~mm}$. The operating principle is based on the deflection of a micro-machined silicon cantilever which reflects the local fluid velocity. Deflection is probed using a superconducting niobium micro-resonator sputtered on the sensor and used as a strain gauge. We present the working principle and the design of the probe, as well as calibration measurements and velocity spectra obtained in a turbulent helium flow above and below the superfluid transition.
\end{abstract}

PACS numbers: 47.80.Fg, 07.10.Cm, 07.10.Pz, 07.57.Kp, 07.20.Mc

Keywords: Cryogenic Helium, Anemometry, Instrumentation

\section{INTRODUCTION}

Cryogenic helium is a fluid of high interest for hydrodynamics experiments because the control parameters (eg. Reynolds number, Rayleigh number, etc.) can be varied over decades and can reach much higher values than most room temperature laboratory experiments [1-5]. One of the main challenges for the experimentalist is then to come up with fast micro-sensors suited to helium flows. Over the last two decades, there has been various developments of local cryogenic probes, specifically hot wires $[2,6,7]$, micro-thermometers $[8,9]$, second-sound tweezers [10], Pitot-tube-like anemometers $[11,12]$, vibrating wires $[13,14]$ and quartz tuning forks $[15,16]$.

When liquid helium is cooled below $T_{\lambda} \approx 2.17 \mathrm{~K}$ (at saturated vapor pressure), it undergoes a phase transition. The classical phase, above $T_{\lambda}$, is called He I. It is a classical fluid with finite viscosity. The phase obtained for temperature below $T_{\lambda}$, is called He II. It can be described by the so-called two-fluid model, ie. as a superposition of a normal component which behaves like a classical fluid (finite viscosity) and a superfluid one with zero-viscosity and quantized vorticity [17].

The smallest, fastest and most widely used local anemometers in turbulence studies are hot-wires. However it is not straightforward to use these probes below the superfluid transition. Indeed, heat is transported

*Present address: Laboratoire de physique, ENS Lyon - 46 allée d'Italie - 69364 Lyon cedex 7, FR very efficiently in the bulk of liquid He II by propagating temperature waves $[18,19]$ and the temperature is roughly homogeneous. While this property of efficient heat transport has proven useful for applications of superfluid helium as a coolant (for example to cool large superconducting coils [20]), it is a major pitfall for temperature-sensitive probes such as hot-wires. So far, the best replacement for velocity measurements has been miniature Pitot tubes produced from cryogenic pressure transducers using dedicated nozzles with external diameters down to $0.6 \mathrm{~mm}$ [12]. Yet for turbulence applications, where smaller probes are needed to resolve the dissipative scales, this approach seems to have nearly reached its limit. Indeed, while the probing of smaller scales requires higher sensibility (smaller eddies are less energetic), reducing the size of a pressure transducer means reducing the diameter of the sensitive membrane and therefore its sensitivity. The alternative method presented in this paper consists in replacing the sensitive membrane by a cantilevered beam. Since it is anchored at only one end, the deflection for a given load is larger than for a membrane of the same size. This leads to higher sensitivity and enable further miniaturization. Other advantages of this type of probes over hot-wires or Pitot tubes are that it measures velocity magnitude and direction and is more immune to acoustic noise than Pitot tubes[36]. Additionnally, the time response of Pitot tubes is limited by a dead volume of fluid. Cantilevers do not suffer from this drawback.

This cantilever-sensing approach has long proven fruitful for atomic force microscopy. More recently, Barth, et al. demonstrated the use of cantilevers as accurate 
anemometers for room-temperature turbulence measurements in air and water[21, 22]. This room-temperature cantilever anemometer uses optical means to accurately measure the beam deflection. This optical approach cannot be brought to cryogenic environment easily. Instead, we present an original method to measure fast fluctuations of the strain on the beam. It is based on a recent technological breakthrough originally aimed at cosmic particles detection and high sensitivity millimeter-wave imaging of the cosmic microwave background.

There is a number of cryogenic hydrodynamic problems that may benefit from this kind of anemometer. (i) The understanding of superfluid turbulence suffers from the lack of spatially-resolved fluctuation data [17]. (ii) In thermal convection, hot-wire measurements are biased by thermal plumes. A reliable local anemometer is still lacking. (iii) The boundary layer thickness in turbulent helium flows is usually small, smaller than $100 \mu \mathrm{m}$ in most cases. Therefore smaller probes are necessary to study it.

The aim of this paper is to discuss the working principle, the details of the construction and driving electronics of a cantilever anemometer specifically designed for superfluid helium.

\section{WORKING PRINCIPLE}

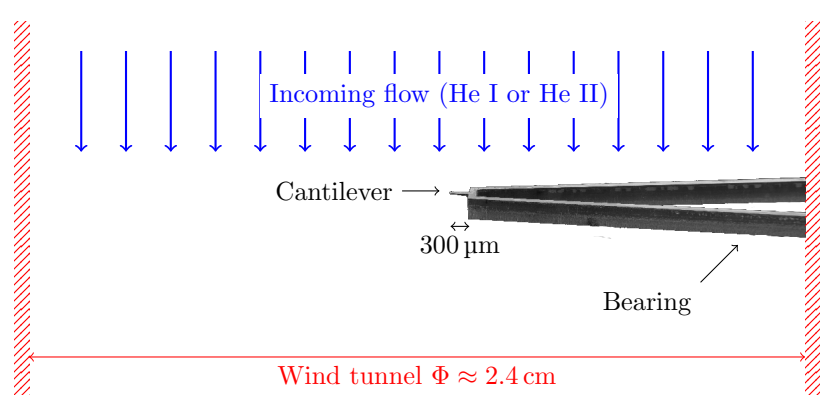

FIG. 1: Cantilever anemometer in the bulk of a liquid helium flow.

The sensitive part of this anemometer is a $100 \mu \mathrm{m} \times 300 \mu \mathrm{m}$ silicon cantilever of thickness between $1 \mu \mathrm{m}$ at the tip and $10 \mu \mathrm{m}$ near the base. A measurement of the strain induced by the incoming flow on this cantilever leads to an estimate of the local velocity (see figure 1). If the fluid comes from the opposite direction, the load is applied on the opposite side of the cantilever: a strain measurement therefore allows an estimate for the velocity magnitude and direction. The deflection $s$ can be estimated using the same hypothesis as in the room temperature case[21],

$$
s=\operatorname{sign}(v) \frac{\ell^{3}}{8} \frac{P \ell w}{E I}
$$

where $\operatorname{sign}(v)= \pm 1$ depending on the flow direction, $\ell$ is the length on the beam, $w$ its width, $e$ its thickness,
$P$ is the applied uniform pressure due to the incoming flow, $E$ is the Young modulus of silicon (its value spans from $130 \mathrm{GPa}$ to $170 \mathrm{GPa}$ depending on the crystal axis, it does not change significantly when the temperature is decreased) and $I=\frac{1}{12} w e^{3}$ is the inertial momentum. Our probe is sensitive to the relative elongation (or relative contraction if $v<0$ ) of the upper surface, that can be derived from Eq. 1,

$$
\frac{\Delta \ell}{\ell} \sim \operatorname{sign}(v) \frac{P}{E} \frac{\ell^{2}}{e^{2}}
$$

The pressure load on the beam due to the flow can be estimated as,

$$
P=\frac{1}{2} c_{d}(v) \rho v^{2}
$$

where $c_{d}(v)$ is the drag coefficient and $\rho \simeq 145 \mathrm{~kg} / \mathrm{m}^{3}$ the fluid density. Above the superfluid transition, the typical Reynolds number of the probe, $R_{\ell}=\ell\langle v\rangle / \nu \approx$ 100 to $10^{4}$ in the velocity range $\langle v\rangle \approx 0.01 \mathrm{~m} / \mathrm{s}$ to $1 \mathrm{~m} / \mathrm{s}$. Therefore, the classical drag coefficient, $c_{d}$ is of order 1 and nearly constant [23]. Experiments suggest that drag forces in turbulent superfluid helium are similar to those in the classical case $[16,24,25]$. Therefore, it seems reasonable to take $c_{d}(v)=O(1)$ to compute a rough estimate of the relative elongation, here for $\langle v\rangle=1 \mathrm{~m} / \mathrm{s}$,

$$
\frac{\Delta \ell}{\ell} \approx 5 \times 10^{-5}
$$

This relative elongation is rather small and therefore always in the linear regime.

To perform fast and sensitive measurements of the deflection of the beam, an accurate strain gauge is needed. Our detection method is based on a superconducting micro-resonator. This technology was initially aimed at astrophysics applications [26-28]. It consists in a lumped-element high-quality-factor radiofrequency micro-resonator patterned in a thin superconducting layer[29, 30] sputter-deposited on the silicon substrate. In the case of astrophysics detectors, absorbed particles (cosmic particles or microwave photons) lead to the breaking of Cooper-pairs and thus to a shift in the resonator kinetic inductance[37] and therefore to a measurable shift of its resonance frequency.

In this work, we demonstrate another possible use of this highly sensitive resonators as strain gauge. The resonator geometry and the superconducting material (200 nm-thick sputter-deposited niobium layer) are chosen such that the kinetic inductance is much lower than the geometric inductance, so that unavoidable cosmic particles do not produce spurious noise. The lumpedelement radiofrequency circuit consists in a LC pattern in which the inductance and the capacitance parts are separated. The inductance is obtained with a meandering pattern, placed on the probe Si chip but outside the flow. This part of the impedance does not depend on the flow velocity. The capacitance originates from interdigitated fingers located on the cantilevered beam. This 
part of the impedance is modified when the beam gets elongated. The full niobium pattern is shown in figure 2 . As a consequence, the relative elongation of the cantilever is designed to result in a measurable shift in resonance frequency.

\section{DESIGN AND OPERATION}

The micro-device - cantilever and bearing arms is patterned from a $350 \mu \mathrm{m}$-thick $\langle 100\rangle$ silicon wafer with double-side photolithography and subsequent deepreactive-ion-etching (DRIE) in $\mathrm{SF}_{6} / \mathrm{O}_{2} / \mathrm{C}_{4} \mathrm{~F}_{8}$ plasma (Bosch process). This lets us choose the geometry of both the sensitive part and the bearing arms to make them rigid and as little invasive as possible for the incoming flow. There are two arms to ensure lateral rigidity. The double side mask is realized with a $400 \mathrm{~nm}$ evaporated aluminum layer. This aluminum is removed at the end of the machining process with a $\mathrm{H}_{3} \mathrm{PO}_{4} / \mathrm{CH}_{3} \mathrm{CO}_{2} \mathrm{H} / \mathrm{H}_{2} \mathrm{SO}_{4}$ liquid mixture.

The use of a barrier layer $-\mathrm{SiO}_{2}$ or $\mathrm{Si}_{3} \mathrm{~N}_{4}$ typically - to control the thickness of the cantilever had to be avoided because the presence of an amorphous dielectric layer is known to produce unwanted spurious frequency and phase noise on superconducting resonators [31, 32]. It is then a technical challenge to leave a $1 \mu \mathrm{m}$-thick cantilever from a $350 \mu \mathrm{m}$ bulk silicon substrate. However, one advantage is that the obtained cantilever is made of mono-crystalline silicon and thus does not hold residual stresses.

To solve this problem, we etched $1 \mu \mathrm{m}$-deep holes in the wafer top side beforehand (see figure 3 ). This leads to a detectable leak between both sides of the wafer during the etching process. When the cantilever tip, being etched from the bottom side, reaches its desired thickness, the gaseous helium cooling jet leaks into the DRIE plasma chamber, which is detected by the pressure regulation systems. The actual cantilever thickness can be estimated from SEM pictures such as the one shown in figure 2 by counting the alternating black and white stripes (called "scallops") on the cantilever side. These are due to the Bosch process alternating cycles. Each cycle roughly etches $1 \mu \mathrm{m}$ in the silicon substrate. The obtained cantilever is slightly less than $1 \mu \mathrm{m}$ at its tip and close to $10 \mu \mathrm{m}$ close to the anchor. This inhomogeneity helps to concentrate the sensitivity to the flow velocity on the tip of the cantilever.

The superconducting micro-resonator is patterned on a $200 \mathrm{~nm}$ niobium layer sputter deposited on the silicon substrate. We use UV-photolithography and subsequent reactive-ion etching in a $\mathrm{SF}_{6} / \mathrm{O}_{2}$ plasma. Niobium was chosen for its ease of use and its relatively high $T_{c}$ of $\sim 9 \mathrm{~K}$.

The micro-resonator is coupled to a coplanar waveguide, called "RF feedline". To sense the resonance frequency of the resonator, a microwave is sent in this waveguide at the port labeled "RF in" and probed at the exit at the port labeled "RF out" (see figure 2). A microwave power between $-30 \mathrm{dBm}$ and $-20 \mathrm{dBm}$ was chosen to get as much output power as possible without weakening the resonance. When the wave frequency matches the LC resonance frequency, part of its energy is transferred to the resonator and the amplitude of the transmitted wave decreases. The coplanar waveguide can be described by a transfer matrix $S$. In the following, we only measure and discuss the transmission function, $\left|S_{21}\right|$, where 2 represents the "RF out" port and 1 the "RF in" port. This function reaches a minimum when the input wave frequency matches the LC resonance frequency, around $1 \mathrm{GHz}$ in our case.

The absorption peak is shown in figure 4 . The coupling strength, and thus the resonance features (depth and width) can be tuned by adjusting the distance between the $50 \Omega$-coplanar waveguide and the meandering pattern inductance (in our case, $18 \mu \mathrm{m}$ overall). The observed resonance is asymmetrical, probably because of the parasitic inductance of the aluminum bondwires, known to be enhanced when the coupling between the feedline and the resonator is strong[29]. The quality factor is large enough for the present application. Yet, it is relatively weak - around $1 \mathrm{~dB}$ deep. The most likely reason is the presence of residual traces of aluminum, passivated by the high-power fluoride plasma during the DRIE steps. Their presence on the niobium circuit has been confirmed by energy-dispersive X-ray spectroscopy analysis. Therefore, it is possible to improve the probe sensitivity further if additional cleaning steps are devised to remove those spurious traces of aluminum.

A measurement consists in evaluating the transmission $S_{21}$ at a given frequency, close to the absorption peak. When the cantilever gets deflected because of the incoming flow, the LC resonance frequency is modified and the output power changes (as illustrated in figure 4). To probe velocity fluctuations, dynamic measurements of the amplitude $\left|S_{21}(t)\right|$ have to be carried out[38]. To do this with a large signal-to-noise ratio, we use a frequency modulation ("FM") measurement technique. The FM wave is sent in the niobium coplanar waveguide. The output wave is amplified at low-temperature with a lownoise cryogenic RF amplifier (Miteq AFS3) located in the vapor of the helium bath of the cryostat, on its lowest thermal screen (the amplifier's casing temperature is close to the liquid helium bath temperature) and recombined with the initial wave using a mixer at room temperature. The amplitude-modulated signal is then demodulated with a lock-in amplifier. The full electronic diagram is shown in figure 5.

\section{IN-FLOW VALIDATION}

The probe, shown in figure 2 , was placed in a cryogenic wind tunnel. It consists in a He II loop continuously powered by a centrifugal pump. Turbulence is probed in a $\Phi=23 \mathrm{~mm}$ diameter, $250 \mathrm{~mm}$ long stainless steel 


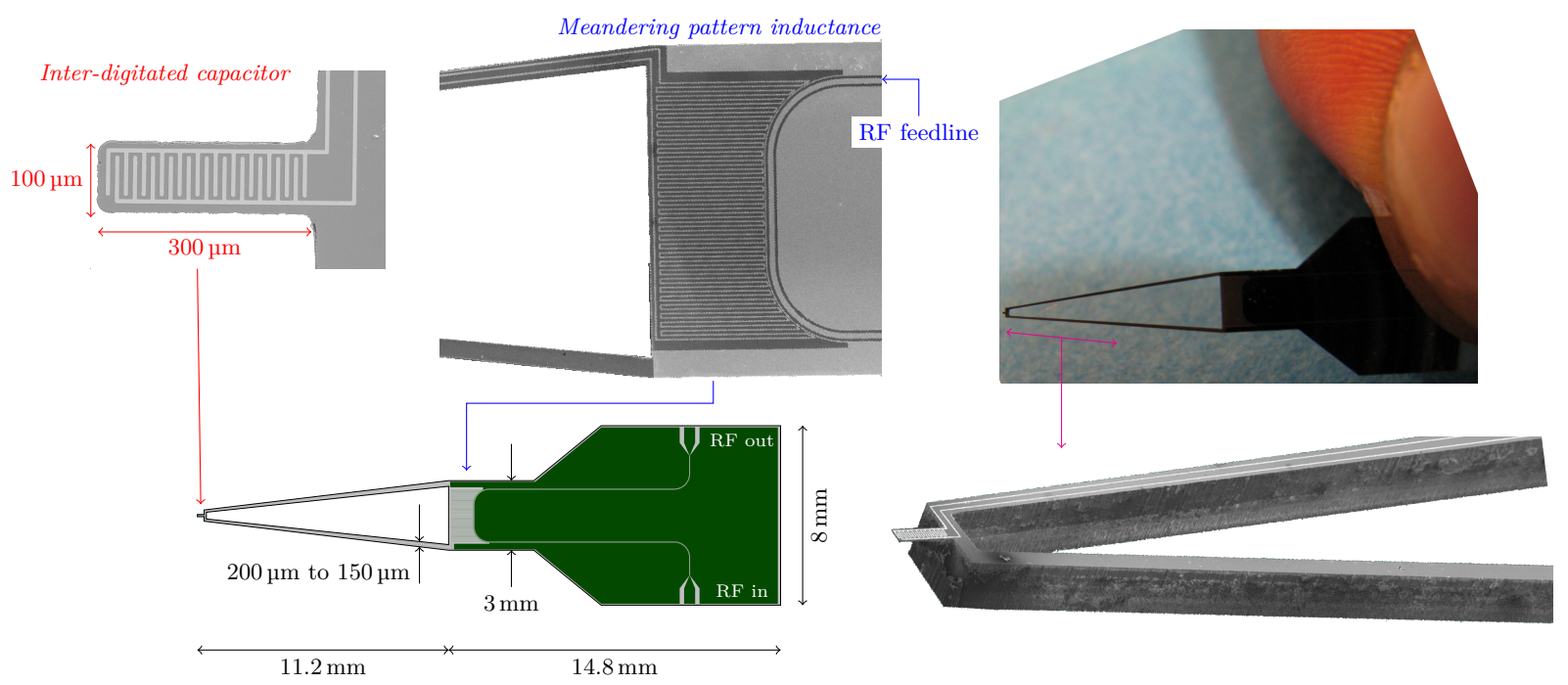

FIG. 2: [Color online] Main dimensions of the probe and niobium pattern of the micro-resonator circuit. The three grayscale images are SEM pictures.

pipe, located upstream the pump. On the return path and at the entrance of the pipe, honeycombs break the spin motion generated by the pump. This cryostat was described in more details in a previous paper [10].

In the present flow set-up, we have added a centered $2.0 \mathrm{~mm}$-thick brass disc with diameter $\varnothing_{d}=11 \mathrm{~mm}$ at the entrance of the pipe which acts as wake generator. The cantilever probe is located $10 \mathrm{~cm}$ downstream from the disc. This disc has the same proportions as the one we used recently in a far-wake superfluid turbulent flow experiment in a larger wind tunnel [33], which showed

a)
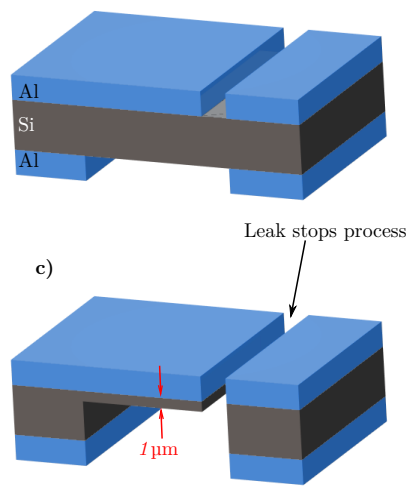

FIG. 3: Schematic description of the deep-etching steps. (a) Bulk silicon wafer with aluminum masks on both sides. (b) $1 \mu \mathrm{m}$-deep RIE etching of top side. (c) Deep-etching of bottom side until a leak is detected. (d) Final structure after cleaning the aluminum layers.

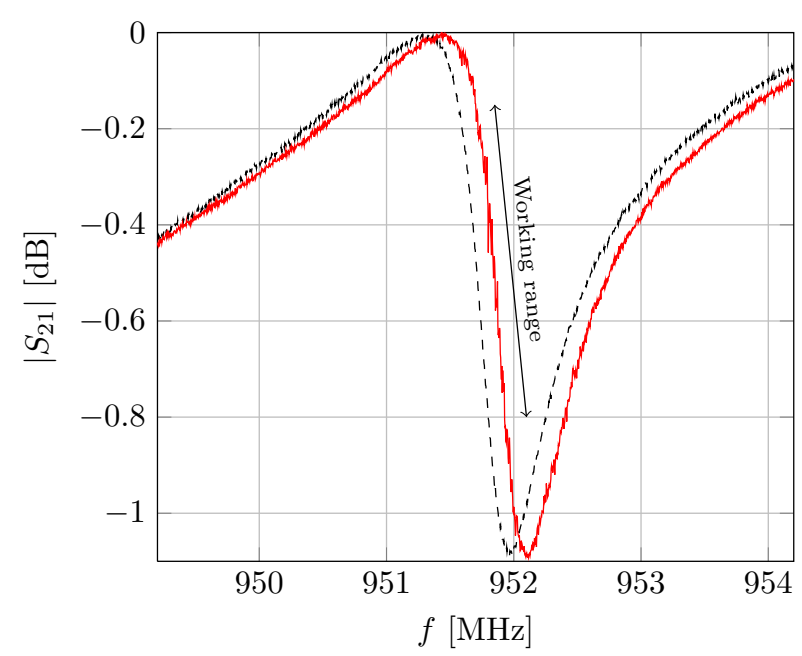

FIG. 4: Transmission coefficient $S_{21}$ through the superconducting feedline (see text) measured with a Vector Network Analyser at $T=1.67 \mathrm{~K}$. The input power on the feedline is $-20 \mathrm{dBm}$. The resonance frequency of this LC-resonator is $952 \mathrm{MHz}$. The quality factor is $Q \approx 10^{3}$. The fluid mean velocity is either zero (black dashed line) or $\approx 1 \mathrm{~m} / \mathrm{s}$ (red line). The "working range" corresponds to the frequency range in which the carrier frequency is chosen when the resonator is probed by frequency modulation (see figure 5).

that the Strouhal number, in such a configuration,

$$
S t=\frac{f_{0} \varnothing_{d}}{\langle v\rangle}
$$

is similar above and below the superfluid transition, where $f_{0}$ is the frequency of the observed vortex shedding and $\langle v\rangle$ is the flow velocity average over the pipe 


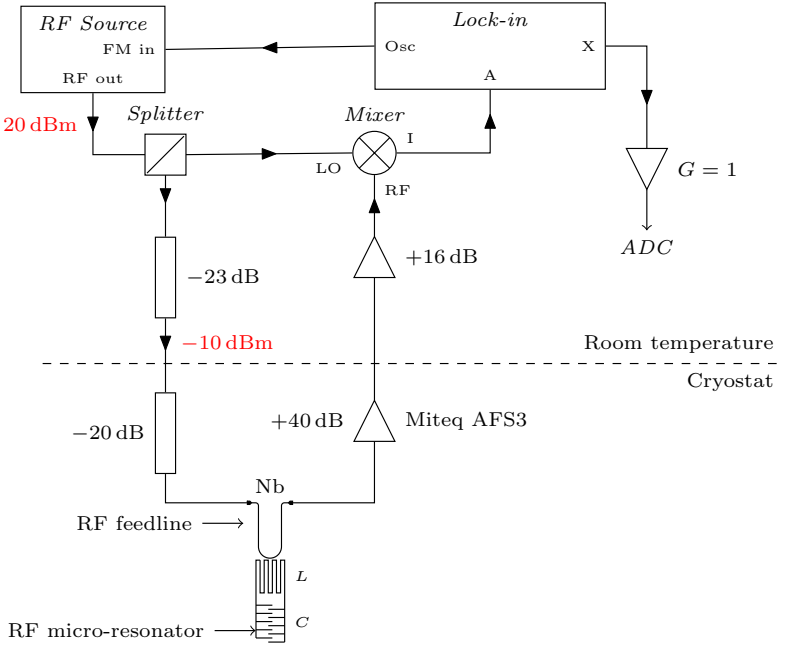

FIG. 5: Electronic diagram used to perform the fluctuations measurements of the cantilever elongation by frequency modulation.

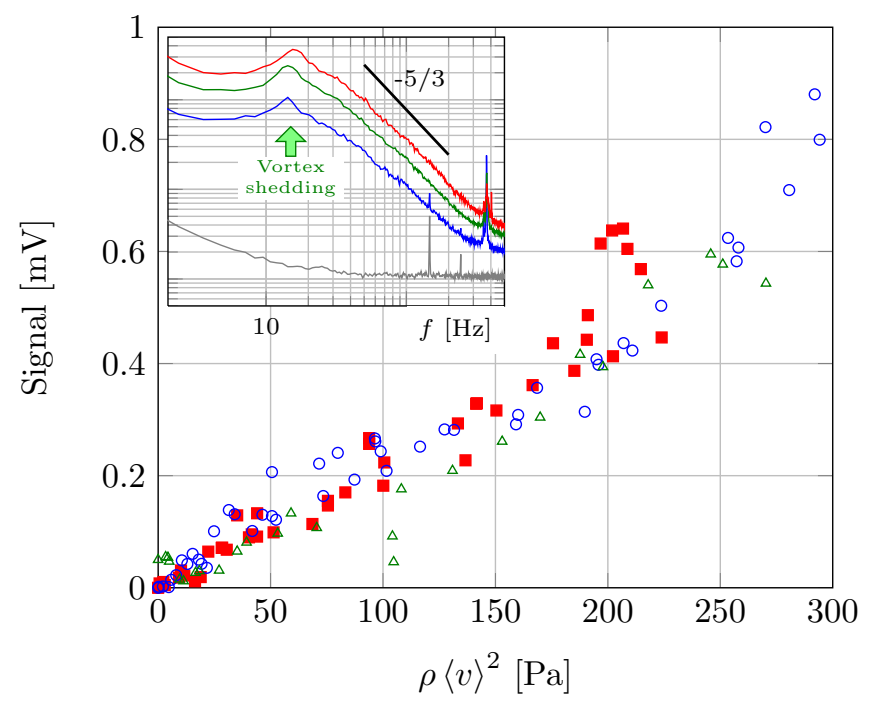

FIG. 6: Calibration of the probe in He II. Open green triangles: $T=2.07 \mathrm{~K}\left(\rho=145.79 \mathrm{~kg} / \mathrm{m}^{3}\right)$. Red squares: $T=$ $1.82 \mathrm{~K}\left(\rho=145.43 \mathrm{~kg} / \mathrm{m}^{3}\right)$. Open blue circles: $T=1.66 \mathrm{~K}$ $\left(\rho=145.31 \mathrm{~kg} / \mathrm{m}^{3}\right)$. Insert: Power specta of the fluctuating signal. From bottom up: $0 \mathrm{~m} / \mathrm{s}$ and $1 \mathrm{~m} / \mathrm{s}$ at $T=1.66 \mathrm{~K}$, $1.1 \mathrm{~m} / \mathrm{s}$ and $1.35 \mathrm{~m} / \mathrm{s}$ at $T=1.82 \mathrm{~K}$. The green arrow points toward the vortex shedding peak.

section.

The mean velocity in the wind tunnel can be varied up to $1.35 \mathrm{~m} / \mathrm{s}$, corresponding to a Reynolds number based on the Taylor microscale $R_{\lambda} \approx 1000$ in He I. The temperature can be varied above and below the superfluid transition at $\approx 2.17 \mathrm{~K}$. The turbulence is probed in the far-wake of the disc.

The cantilever probe is calibrated against a minia- ture Pitot probe, at several temperatures. The raw data points in He II are shown in figure 6 for temperature from $1.66 \mathrm{~K}$ to $2.07 \mathrm{~K}$, which corresponds to concentration of superfluid component, $\rho_{s} / \rho$, ranging from $80 \%$ to $30 \%$ respectively. At each temperature, the calibration dataset is obtained by stepwise increase and decrease of the rotation speed of the wind-tunnel propeller. Each step includes a 20-seconds pause allowing the hydrodynamical and thermal transient behaviours to relax, followed by a 3 -seconds averaging of the cantilever probe signal. The scatter in the calibration plot (see figure 6) is interpreted as the result of the fluctuations in the windtunnel (residual transient motions and turbulent fluctuations) and not to the intrinsic noise of the probe, nor from electronic noises. Two types of measurements support this interpretation. First, the intrinsic noise of the electronics is measured in quiescient superfluid helium. The spectral density of the corresponding noise signal, plotted in the insert of figure 6 (lower curve), is significantly lower than the spectral density of the signal in presence of a flow. The second test - presented in the next paragraph - consists in comparing the fluctuations spectra measured by the cantilever probe with the expected turbulent velocity spectra. The calibration in He I was more difficult because of the sporadic presence of bubbles in this specific wind tunnel. We find that the obtained signal scales like $\rho\langle v\rangle^{2}$ to a good approximation and do not seem to depend on temperature, as expected (see Eq. 2 and 3).

The power spectra of the signal, obtained for several superfluid turbulent conditions, are shown in insert of figure 6. They exhibit expected hydrodynamic features [33]: (i) a low frequency plateau up to $20 \mathrm{~Hz}$ corresponding to scales larger than the injection scale, (ii) a vortex shedding peak near $15 \mathrm{~Hz}$ and (iii) nearly a decade of $f^{-5 / 3}$ Kolmogorov scaling corresponding to inertial scales of the turbulent cascade.

\section{CONCLUSION \& PERSPECTIVES}

We have realized and operated the first cantilever anemometer dedicated to cryogenic turbulence and the first fully micromachined local probe for cryogenic hydrodynamics. The calibration and turbulent fluctuations measurements validate the principle of cantilever anemometry for superfluid flows. This technology improves by a factor 10 the spatial resolution compared to the best alternative anemometers known to work in superfluid helium.

The cantilever approach has several advantages over traditional pressure-transducer based anemometers and supersedes Pitot tube probes in many aspects: (i) it is fully micro-machined, which opens the way to further miniaturization down to scales where quantum effects are supposedly occurring in superfluid turbulence at finite temperature[33], (ii) it is mostly immune to acoustic perturbations which is one of the main noise sources 
in high-Reynolds number superfluid wind tunnels, (iii) it provides the sign of the velocity and (iv) the spurious heat dissipation is negligible thanks to the superconducting technology (below $1 \mu \mathrm{W}$ ). This is a major advantage for thermal convection systems.

This work also demonstrates the interest and efficiency of superconducting micro-resonators for fine hydrodynamic measurements. Since the resonance frequency depends on the dielectric permittivity of the fluid[30] and therefore on the local density (and $\rho(T) \propto T$ ), this opens the way to other possible use in cryogenic turbulence, such as local density or temperature measurements. Additionally, it was shown previously that large numbers of these micro-resonators can be multiplexed on a single feedline[28], which allows to build arrays of cantilevers and therefore grants access to spatial velocity correlations. These multiplexed superconducting resonators can also be deposited on a surface to obtain an image of its temperature. One may think for example to realize ther- mal imaging of the boundary layer in cryogenic convection experiments where the heat flux spatial behavior is an important open question directly related to the ultimate regime of convection[34, 35].

\section{Acknowledgements}

This work was performed with the help of "Plateforme Technologie Amont" (PTA) in Grenoble and with financial support of the ANR (grants "SHREK" ANR09-BLAN-0094 and "MKIDS" ANR-09-JCJC-0021-01). We are grateful to T. Haccart, S. Litaudon, F. Gustavo, C. Lemonias and J.-L. Thomassin for their expertise in the clean room. We thank G. Garde for his availability and skills on the cryogenic apparatus, and C. Thirion, C. Hoarau, V. Bouchiat and B. Hébral for many stimulating discussions.
[1] O. Chanal, B. Chabaud, B. Castaing, and B. Hébral, Eur. Phys. J. B 17, 309 (2000).

[2] S. Pietropinto, C. Poulain, C. Baudet, B. Castaing, B. Chabaud, Y. Gagne, B. Hébral, Y. Ladam, P. Lebrun, O. Pirotte, et al., Physica C 386, 512 (2003).

[3] P.-E. Roche, F. Gauthier, R. Kaiser, and J. Salort, New J. Phys. 12, 085014 (2010).

[4] J. J. Niemela, L. Skrbek, K. R. Sreenivasan, and R. J. Donnelly, Nature 404, 837 (2000).

[5] P. Urban, P. Hanzelka, T. Kralik, V. Musilova, L. Skrbek, and A. Srnka, Rev. Sci. Instrum. 81, 085103 (2010).

[6] B. Castaing, B. Chabaud, and B. Hébral, Rev. Sci. Instrum. 63, 4167 (1992).

[7] J. Maurer, P. Tabeling, and G. Zocchi, EPL 26, 31 (1994).

[8] O. Chanal, B. Baguenard, O. Béthoux, and B. Chabaud, Rev. Sci. Instrum. 6, 2442 (1997).

[9] F. Gauthier, J. Salort, O. Bourgeois, J.-L. Garden, R. du Puits, A. Thess, and P.-E. Roche, EPL 87, 44006 (2009).

[10] P.-E. Roche, P. Diribarne, T. Didelot, O. Français, L. Rousseau, and H. Willaime, EPL 77, 66002 (2007).

[11] J. Maurer and P. Tabeling, EPL 43, 29 (1998).

[12] J. Salort, P. Diribarne, B. Rousset, Y. Gagne, B. Dubrulle, T. Didelot, B. Chabaud, F. Gauthier, B. Castaing, and P.-E. Roche, Phys. fluids 22, 125102 (2010).

[13] D. I. Bradley, D. O. Clubb, S. N. Fisher, A. M. Guénault, R. P. Haley, C. J. Matthews, G. R. Pickett, and K. L. Zaki, J. Low Temp. Phys. 138, 493 (2005).

[14] Y. Nago, T. Ogawa, K. Obara, H. Yano, O. Ishikawa, and T. Hata, J. Low Temp. Phys. 162, 322 (2011).

[15] R. Blaauwgeers, M. Blažková, M. Človečko, V. B. Eltsov, R. de Graaf, J. Hosio, M. Krusius, D. Schmoranzer, W. Schoepe, L. Skrbek, et al., J. Low Temp. Phys. 146 (2007).

[16] D. Schmoranzer, M. L. Mantia, and L. Skrbek, EPJ Web of Conferences 25, 02025 (2012).

[17] L. Skrbek and K. R. Sreenivasan, Phys. fluids 24, 011301
(2012).

[18] L. Landau, Phys. Rev. 60, 356 (1941).

[19] S. V. Sciver, Helium Cryogenics (Springer, 1986), ISBN 978-0-306-42335-2.

[20] P. Lebrun and L. Tavian, in CAS - CERN Accelerator School on Superconductivity and Cryogenics for Accelerators and Detectors (2002), pp. 375-389.

[21] S. Barth, H. Koch, A. Kittel, J. Peinke, J. Burgold, and H. Wurmus, Rev. Sci. Instrum. 76, 075110 (2005).

[22] M. Hölling and J. Peinke, Springer Proc. in Phys. 131, 61 (2009).

[23] R. L. C. Flemmer and C. L. Banks, Powder Technology 48, 217 (1986).

[24] M. R. Smith, D. K. Hilton, and S. W. V. Sciver, Phys. fluids 11, 751 (1999).

[25] M. Blažková, D. Schmoranzer, and L. Skrbek, Phys. Rev. E 75, 025302(R) (2007).

[26] P. K. Day, H. G. LeDuc, B. A. Mazin, A. Vayonakis, and J. Zmuidzinas, Nature 425, 817 (2003).

[27] B. A. Mazin, Ph.D. thesis, California Institute of Technology (2004).

[28] A. Monfardini, L. Swenson, A. Bideaud, F. Désert, S. Yates, A. Benoit, A. Baryshev, J. Baselmans, S. Doyle, B. Klein, et al., Astronomy and Astrophysics 521, A29 (2010).

[29] S. Doyle, P. Mauskopf, J. Naylon, A. Porsch, and C. Duncombe, J. Low Temp. Phys. 151, 530 (2008).

[30] G. Grabovskij, L. Swenson, O. Buisson, C. Hoffmann, A. Monfardini, and J.-C. Villégier, Applied Physics Letters 93, 134102 (2008).

[31] S. Kumar, J. Gao, J. Zmuidzinas, B. A. Mazin, H. G. LeDuc, and P. K. Day, Applied Physics Letters 92, 123503 (2008).

[32] R. Barends, H. L. Hortensius, T. Zijlstra, J. J. A. Baselmans, S. J. C. Yates, J. R. Gao, and T. M. Klapwijk, Applied Physics Letters 92, 223502 (2008).

[33] J. Salort, B. Chabaud, E. Lévêque, and P.-E. Roche, EPL 97, 34006 (2012).

[34] F. Gauthier and P.-E. Roche, EPL 83, 24005 (2008). 
[35] F. Chillà and J. Schumacher, Eur. Phys. J. E 35, 58 (2012).

[36] An acoustic perturbation would be sensed equally by both sides, at first order

[37] The inertia of the superconducting, lossless charge carriers, i.e. the Cooper pairs, leads to an equivalent in- ductance term called "kinetic inductance", eg. see: V. V. Schmidt, The physics of Superconductors (Springer, Berlin, 1997)

[38] An alternative approach is to measure the phase of $S_{21}$ instead of its amplitude. 